\title{
A free boundary problem arising in a simplified tumour growth model of contact inhibition
}

\author{
M. BERTSCH \\ Istituto per le Applicazioni del Calcolo Mauro Picone, CNR, Via dei Taurini 19, 00185 Roma, Italy; \\ Dipartimento di Matematica, Università di Roma Tor Vergata, 00133 Roma, Italy \\ E-mail:m.bertsch@iac.cnr.it \\ R. DAL PASSO ${ }^{\dagger}$ \\ Dipartimento di Matematica, Università di Roma Tor Vergata, 00133 Roma, Italy \\ M. MIMURA \\ Meiji Institute for Advanced Study of Mathematical Sciences, Meiji University, \\ 1-1-1, Higashimita, Tamaku, Kawasaki, 214-8571, Japan
}

[Received 1 October 2009 and in revised form 4 April 2010]

\begin{abstract}
It is observed in vitro and in vivo that when two populations of different types of cells come near to each other, the rate of proliferation of most cells decreases. This phenomenon is often called contact inhibition of growth between two cells. In this paper, we consider a simplified 1-dimensional PDEmodel for normal and abnormal cells, motivated by the paper by Chaplain, Graziano and Preziosi ([5]). We show that if the two populations are initially segregated, then they remain segregated due to the contact inhibition mechanism. In this case the system of PDE's can be formulated as a free boundary problem.
\end{abstract}

\section{Introduction}

It is observed in vitro and in vivo that when two different types of cells approach closely, the rate of proliferation of most cells decreases [1]. This phenomenon is often called contact inhibition of growth between two cells and is observed in many types of cells. For a better theoretical understanding of the mechanism of contact inhibition arising in normal and abnormal (tumour) tissue growth, several mathematical models have been proposed so far. Among them, we mention the model proposed by Chaplain, Graziano and Preziosi ([5]) which describes normal and abnormal tissue growth. Here the "abnormal cells" denote cells which respond to compression in a different way from the normal cells. According to the phenomenological description given by biologists, it is believed that these cells change to be a tumour at some later stage in the process. The feature of their model includes the effect of pushing cells away from the overcrowded region where they feel pressed. In other words, the cells move in the direction of lower overall density of cells.

Here we introduce a simplified version of the model proposed in [5]. Let $n$ and $a$ be the densities of normal and abnormal cells and $N$ be the overall density of cells, $N=n+a$. Then the model for

\footnotetext{
${ }^{\dagger}$ In 2007 Roberta Dal Passo died prematurely. Roberta was an excellent mathematician and she gave a substantial contribution to the present paper.
} 
the two types of cells is given by

$$
\left\{\begin{array}{l}
n_{t}=\nabla(n \nabla V(N))+G_{n}(N) n \\
a_{t}=\nabla(a \nabla V(N))+G_{a}(N) a,
\end{array}\right.
$$

where $V(N)$ is an increasing function in $N$. A possible choice for $V(N)$ is $V(N)=N$, which is proposed in [5]. $G_{n}(N)$ is a nonincreasing function satisfying $G_{n}\left(N_{n}\right)=0$ for some constant $N_{n}>0$. Similarly $G_{a}(N)$ depends on some constant $N_{a}>0$. As a simple example, we take $G_{n}(N)=g_{n}\left(1-N / N_{n}\right)$ and $G_{a}(N)=g_{a}\left(1-N / N_{a}\right)$ for some constants $g_{n}>0$ and $g_{a}>0$, which are ecologically regarded as a Lotka-Volterra competition type. For (1), the following questions arise naturally:

(Q1) Let the two types of cells be initially segregated. When they meet, do they form a barrier for each other, i.e. do they never overlap?

(Q2) Suppose that, as a result of a mutation, the abnormal cells may be generated in a small region of the domain of normal cells. How do the abnormal cells $n$ invade the state of normal cells in their evolution?

Motivated by these questions, we consider system (1) in one spatial dimension. First, we choose $V(N)=N$ and study the following system for $n$ and $a$ numerically:

$$
\begin{cases}n_{t}=\left(n N_{x}\right)_{x}+(1-N) n & \text { for }-L<x<L, t>0, \\ a_{t}=\left(a N_{x}\right)_{x}+(1-N / k) a & \text { for }-L<x<L, t>0 .\end{cases}
$$

Here $k$ is a positive constant satisfying $k>1$. At $x=-L$ and $x=L$ we impose zero-flux boundary conditions.

In the first example we consider question (Q1): $n(0, x)$ and $a(0, x)$ are completely separated. Fig. 1 suggests that when $n$ and $a$ come into contact, they do not overlap for later times. This indicates that the dynamics of $n$ and $a$ can be described by a free boundary problem with a contact point, say $x=\zeta(t)$.

In the second example question $(\mathrm{Q} 2)$ is considered: the initial conditions satisfy $n(0, x)+$ $a(0, x)=1$ for $-L \leqslant x \leqslant L, a(0, x) \equiv 0(x \leqslant-\pi / m$ and $\pi / m \leqslant x)$ and $a(0, x)=$ $a(1+\cos (m x))(-\pi / m \leqslant x \leqslant \pi / m)$. As shown in Fig. 2, the abnormal cells press and invade the state of the normal cells, which qualitatively seems to be in agreement with the biological observations. In addition, Fig. 2 suggests that for large times the solutions behave as (almost) segregated solutions.

These two numerical experiments lead naturally to quite a few questions. In this paper we begin considering basic questions concerning the correct formulation and wellposedness of both the free boundary problem and a more general system (when solutions do not have separated supports). For this purpose, we shall consider a 1-dimensional system which is more general than (2). More precisely, our goal is to find bounded and nonnegative functions $u(x, t)$ and $v(x, t)(x \in[-L, L]$, $t \geqslant 0$ ) which satisfy, in a sense to be defined,

$$
\begin{cases}u_{t}=\left(u(\chi(u+v))_{x}\right)_{x}+u(1-u-\alpha v) & \text { for }-L<x<L, t>0, \\ v_{t}=d\left(v(\chi(u+v))_{x}\right)_{x}+\gamma v(1-\beta u-v / k) & \text { for }-L<x<L, t>0, \\ (u+v)_{x}( \pm L, t)=0 & \text { for } t>0, \\ u(x, 0)=u_{0}(x), \quad v(x, 0)=v_{0}(x) & \text { for }-L<x<L .\end{cases}
$$



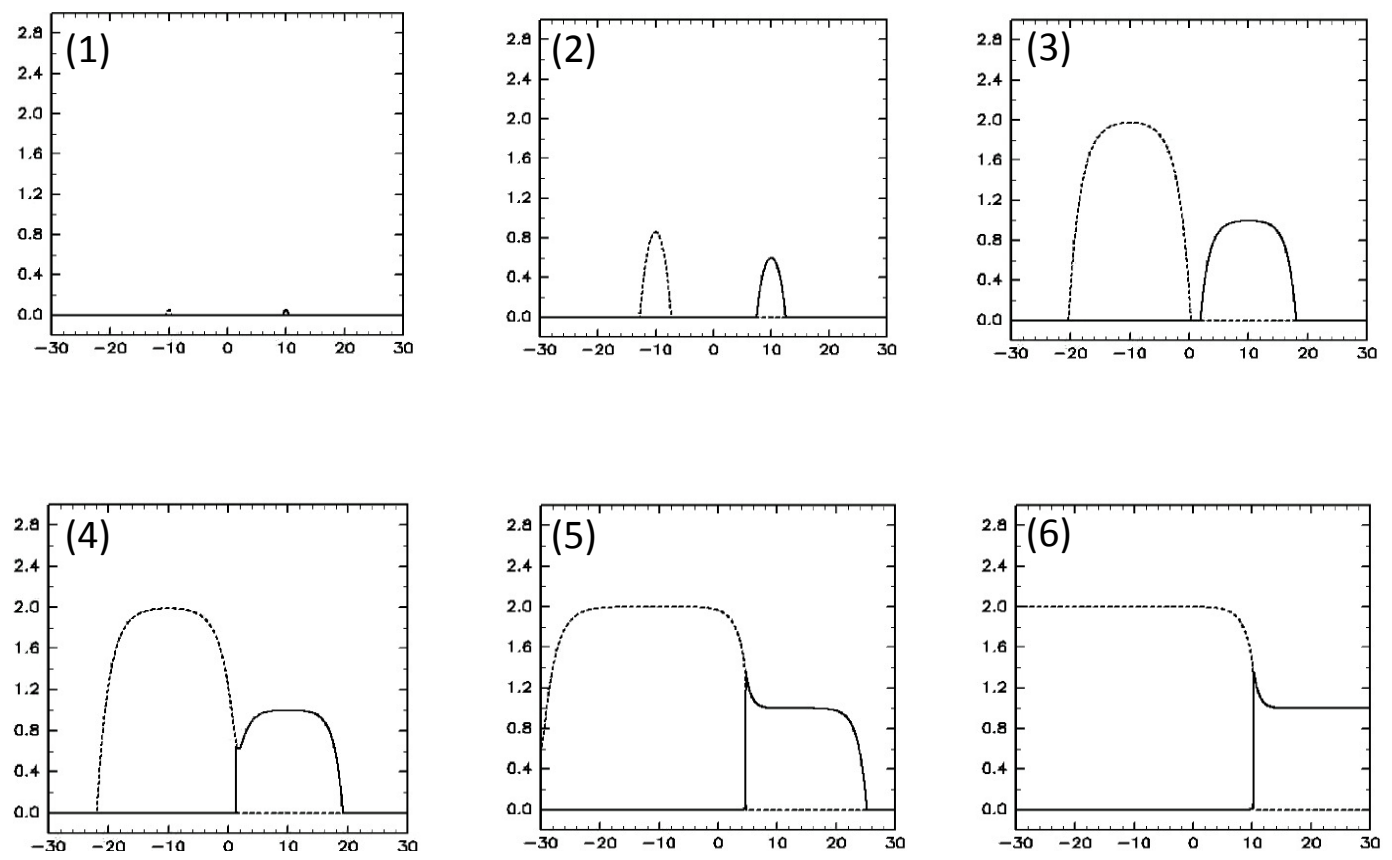

FIG. 1. Contact inhibition of $a(--)$ and $n(-)$ of (2) where $L=30, k=2$
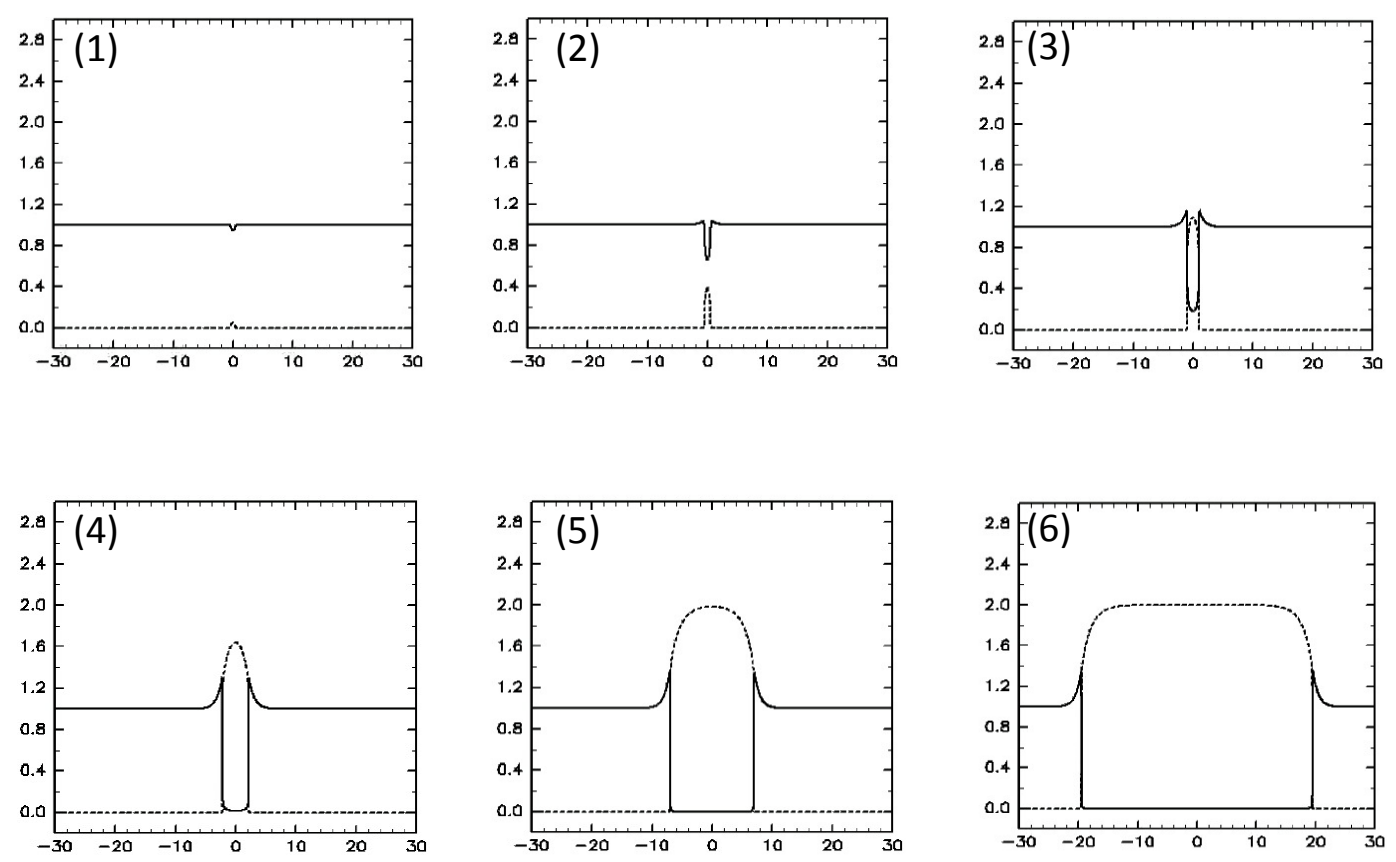

FIG. 2. Invasion of $a(--)$ in the field of $n(-)$ of (2) where $L=30, k=2, m=3$ 
Here $\chi: \mathbb{R}^{+} \rightarrow \mathbb{R}$ is a smooth function such that $\chi^{\prime}>0$ in $\mathbb{R}^{+}, L, d, k, \alpha, \beta$ and $\gamma$ are positive constants and $u_{0}$ and $v_{0}$ are the initial functions, $u_{0}, v_{0} \in L^{\infty}(-L, L)$, satisfying

$$
u_{0}, v_{0} \geqslant 0 \text { and } 0<A_{0} \leqslant u_{0}+v_{0} \leqslant B_{0} \quad \text { a.e. in }(-L, L) .
$$

We can regard (3) as a competition system with inter-specific competition rates $\alpha$ and $\beta$. When $\chi(s)=s$ for $s>0, d=\alpha=\gamma=1$ and $\beta=1 / k,(3)$ obviously reduces to (2).

The strict positivity of $u_{0}+v_{0}$ in (4) has been assumed for the sake of simplicity, and it explains why we do not require anything about the behaviour of $\chi(s)$ near $s=0$. Possible choices of $\chi$ are: $\chi(s)=s^{m}$ or $\chi(s)=-s^{-m}(m>0)$, and $\chi(s)=\log s$ [9]. In terms of question (Q1) the strict positivity of $u_{0}+v_{0}$ means that we study the system after the two types of cells have already met.

So we begin by considering the case that $u$ and $v$ are initially segregated: let $x_{0} \in(-L, L)$ be such that

$$
v_{0}=0 \quad \text { a.e. in }\left(-L, x_{0}\right) \text { and } \quad u_{0}=0 \quad \text { a.e. in }\left(x_{0}, L\right) .
$$

We shall show that there exists a solution $(u, v)$ of problem 3 such that $u(t)$ and $v(t)$ remain segregated for all later times: there exists a continuous function $\zeta:[0, \infty) \rightarrow(-L, L)$ such that

$$
\begin{cases}v(x, t)=0 & \text { for } t>0 \text { and a.e. } x \in(-L, \zeta(t)) \\ u(x, t)=0 & \text { for } t>0 \text { and a.e. } x \in(\zeta(t), L)\end{cases}
$$

More precisely, we shall prove the following result.

TheOREM 1.1 Let $\chi: \mathbb{R}^{+} \rightarrow \mathbb{R}$ be a smooth function such that $\chi^{\prime}>0$ in $\mathbb{R}^{+}$. Let $d, L, k, \alpha, \beta$ and $\gamma$ be positive constants. Let $u_{0}, v_{0} \in L^{\infty}(-L, L)$ and $x_{0} \in(-L, L)$ satisfy (4) and (5). Then there exists a pair of functions $(u, v) \in\left(L^{\infty}((-L, L) \times(0, \infty))\right)^{2}$ which is a solution of problem (3) in the following sense: there exists a function $\zeta:[0, \infty) \rightarrow(-L, L)$ which is continuous in $[0, \infty)$ and of class $C^{1}$ in $(0, \infty)$ such that, if we set

$$
\mathcal{P}_{u}=\left\{(x, t) \in[-L, L] \times \mathbb{R}^{+} ; x<\zeta(t)\right\}, \quad \mathcal{P}_{v}=\left\{(x, t) \in[-L, L] \times \mathbb{R}^{+} ; x>\zeta(t)\right\},
$$

then:

(i) $u, v \in C^{2,1}\left(\mathcal{P}_{u} \cup \mathcal{P}_{v}\right), u>0$ and $v=0$ in $\mathcal{P}_{u}, u=0$ and $v>0$ in $\mathcal{P}_{v}$;

(ii) $u_{t}=\left(u(\chi(u))_{x}\right)_{x}+u(1-u)$ in $\mathcal{P}_{u}, v_{t}=d\left(v(\chi(v))_{x}\right)_{x}+\gamma v(1-v / k)$ in $\mathcal{P}_{v}$;

(iii) $u \in C^{1,0}\left(\mathcal{P}_{u} \cup\{(\zeta(t), t) ; t>0\}\right), v \in C^{1,0}\left(\mathcal{P}_{v} \cup\{(\zeta(t), t) ; t>0\}\right)$;

(iv) $\lim _{x \rightarrow \zeta(t)^{-}} u(x, t)=\lim _{x \rightarrow \zeta(t)^{+}} v(x, t)>0$ if $t>0$;

(v) $\zeta^{\prime}(t)=-\lim _{x \rightarrow \zeta(t)^{-}}(\chi(u))_{x}(x, t)=-\lim _{x \rightarrow \zeta(t)^{+}} d(\chi(v))_{x}(x, t)$ if $t>0$;

(vi) $\zeta(0)=x_{0}$, and $u(t) \rightarrow u_{0}$ and $v(t) \rightarrow v_{0}$ in $L^{1}(-L, L)$ as $t \rightarrow 0^{+}$;

(vii) $u_{x}(-L, t)=0$ and $v_{x}(L, t)=0$ for $t>0$.

In other words, setting

$$
w(x, t)=u(x, t)+v(x, t)= \begin{cases}u(x, t) & \text { if }-L \leqslant x<\zeta(t), \\ v(x, t) & \text { if } \zeta(t)<x \leqslant L\end{cases}
$$

and

$$
w_{0}(x)=u_{0}(x)+v_{0}(x)= \begin{cases}u_{0}(x) & \text { if }-L \leqslant x<x_{0} \\ v_{0}(x) & \text { if } x_{0}<x \leqslant L\end{cases}
$$


the pair $(w, \zeta)$ is a solution of the following free boundary problem:

$$
\begin{cases}w_{t}=\left(w(\chi(w))_{x}\right)_{x}+w(1-w) & \text { if }-L<x<\zeta(t), t>0, \\ w_{t}=d\left(w(\chi(w))_{x}\right)_{x}+\gamma w(1-w / k) & \text { if } \zeta(t)<x<L, t>0, \\ w_{x}( \pm L, t)=0 & \text { for } t>0, \\ w(x, 0)=w_{0}(x) & \text { for a.e. } x \in(-L, L), \\ w\left(\zeta(t)^{-}, t\right)=w\left(\zeta(t)^{+}, t\right) & \text { for } t>0, \\ \zeta^{\prime}(t)=-(\chi(w))_{x}\left(\zeta(t)^{-}, t\right)=-d(\chi(w))_{x}\left(\zeta(t)^{+}, t\right) & \text { for } t>0, \\ \zeta(0)=x_{0} . & \end{cases}
$$

Observe that it is natural to refer to the pair $(u, v)$ as a solution of problem (3). For example, it is easy to check that for any $T>0$ and test function $\varphi \in C^{\infty}([-L, L] \times[0, T])$ the functions $u$ and $v$ defined by Theorem 1.1 satisfy the integral identities

$$
\begin{aligned}
\int_{0}^{T} \int_{-L}^{L} u \varphi_{t} \mathrm{~d} x \mathrm{~d} t= & \int_{0}^{T} \int_{-L}^{L} u(\chi(u+v))_{x} \varphi_{x} \mathrm{~d} x \mathrm{~d} t-\int_{0}^{T} \int_{-L}^{L} u(1-u-\alpha v) \varphi \mathrm{d} x \mathrm{~d} t \\
& +\int_{-L}^{L} u(x, T) \varphi(x, T) \mathrm{d} x-\int_{-L}^{L} u_{0}(x) \varphi(x, 0) \mathrm{d} x
\end{aligned}
$$

and

$$
\begin{aligned}
\int_{0}^{T} \int_{-L}^{L} v \varphi_{t} \mathrm{~d} x \mathrm{~d} t= & \int_{0}^{T} \int_{-L}^{L} d v(\chi(u+v))_{x} \varphi_{x} \mathrm{~d} x \mathrm{~d} t-\int_{0}^{T} \int_{-L}^{L} \gamma v\left(1-\beta u-\frac{v}{k}\right) \varphi \mathrm{d} x \mathrm{~d} t \\
& +\int_{-L}^{L} v(x, T) \varphi(x, T) \mathrm{d} x-\int_{-L}^{L} v_{0}(x) \varphi(x, 0) \mathrm{d} x .
\end{aligned}
$$

In particular we used here property (iv) of Theorem 1.1 Note that in [5], (iv) is a consequence of a constitutive assumption for the stress (expressing the stress continuity).

Although we are primarily interested in the existence of segregated solutions, the numerical results in Fig. 2 suggest a particularly challenging problem: are there parameter values for which the solutions of the free boundary problem are asymptotically stable in the class of non-segregated solutions? A preliminary question is whether problem (3) has a solution if initially $u$ and $v$ are not segregated. In general we are not able to answer this question, but in the case of equal dispersal velocities $(d=1)$ such a solution does exist:

THEOREM 1.2 Let $\chi: \mathbb{R}^{+} \rightarrow \mathbb{R}$ be a smooth function such that $\chi^{\prime}>0$ in $\mathbb{R}^{+}$. Let $L, k, \alpha, \beta$ and $\gamma$ be positive constants and let

$$
d=1 \text {. }
$$

Let $u_{0}, v_{0} \in B V(-L, L)$ be such that $u_{0}+v_{0} \in C^{1}([-L, L]),\left(u_{0}+v_{0}\right)_{x}$ is Hölder continuous in $[-L, L], u_{0} \geqslant 0$ and $v_{0} \geqslant 0$ a.e. in $(-L, L)$, and $u_{0}+v_{0}>0$ in $[-L, L]$. Then there exists a pair of functions $(u, v) \in\left(L^{\infty}((-L, L) \times(0, \infty))\right)^{2}$ which is a solution of problem $(3)$ in the following sense: setting

$$
w:=u+v \quad \text { a.e. in }(-L, L) \times(0, \infty),
$$

we have, for any $T>0$, 
(i) $u, v \in L^{\infty}(0, T ; B V(-L, L)) \cap B V((-L, L) \times(0, T)) \cap C\left(0, T ; L^{1}(-L, L)\right)$ and $u, v \geqslant 0$ a.e. in $(-L, L) \times(0, T)$;

(ii) $w \in C([-L, L] \times[0, T]), w_{x}$ is Hölder continuous in $[-L, L] \times(0, T]$ and $w_{t}, w_{x x} \in$ $L^{2}((-L, L) \times(0, T))$

(iii) for any test function $\varphi \in C^{\infty}([-L, L] \times[0, T]), u$ and $v$ satisfy [10] and [11].

Both theorems were already known in the special case where $\chi(s)=s$ and the equations for $u$ and $v$ do not contain reaction terms, but the proofs ([2], [3], [4]) heavily rely on the fact that without reaction terms the equations reduce to conservation laws. Indeed the main step in the proof of Theorem 1.1 is to find the structure (cf. problem (12) below) which makes it possible to use mass variables even though the equations are not conservation laws. The proof of Theorem 1.2 is mainly based on BV-estimates. The natural generalization of our results to the case of higher spatial dimensions is completely open.

The present paper is a first attempt to understand the mathematical structure of problem (3). In future papers we shall focus on the qualitative behaviour of solutions: existence of travelling wave solutions, their stability, and the special role of segregated solutions.

\section{Existence of segregated solutions}

In this section we prove Theorem 1.1 Suppose that $u, v$ and $\zeta$ have the properties listed in Theorem 1.1, If we set

$$
D(x, t)=\left\{\begin{array}{ll}
1 & \text { if }-L \leqslant x<\zeta(t), \\
d & \text { if } \zeta(t)<x \leqslant L,
\end{array} \quad h(x, t, w)= \begin{cases}1-w & \text { if }-L \leqslant x<\zeta(t), \\
\gamma(1-w / k) & \text { if } \zeta(t)<x \leqslant L,\end{cases}\right.
$$

then the function $w:=u+v$ has the following properties for $t>0$ :

- $w_{t}=\left(D(x, t) w \chi^{\prime}(w) w_{x}\right)_{x}+w h(x, t, w)$ if $x \neq \zeta(t)$

- $w$ and $D \chi^{\prime}(w) w_{x}$ are continuous functions across the free boundary $x=\zeta(t)$

(more precisely, they can be considered as continuous functions by properly (re)defining them at the interface $x=\zeta(t))$.

The free boundary $x=\zeta(t)$ can be considered as a characteristic of the equation for $w: \zeta^{\prime}(t)=$ $-D \chi^{\prime}(w(\zeta(t), t)) w_{x}(\zeta(t), t)$ for $t>0$ (where $D \chi^{\prime}(w) w_{x}$ is the continuous function mentioned before). Therefore it is convenient to introduce a Lagrangian variable $y$, defining the change of variables $(x, t) \mapsto(y, t)$ by the following family of characteristics $x=X(y, t): X$ is the solution of

$$
\begin{cases}X_{t}(y, t)=-D(X(y, t), t) \chi^{\prime}(w(X(y, t), t)) w_{x}(X(y, t), t) & \text { if }-L \leqslant y \leqslant L, t>0 \\ X(y, 0)=y & \text { if }-L \leqslant y \leqslant L .\end{cases}
$$

This change of variables transforms the interface $x=\zeta(t)$ into the vertical line $y=x_{0}$, and $D$ and $h$ become functions depending on $y$ but not on $t$ :

$$
D(y)=\left\{\begin{array}{ll}
1 & \text { if }-L \leqslant y<x_{0}, \\
d & \text { if } x_{0}<y \leqslant L,
\end{array} \quad h(y, w)= \begin{cases}1-w & \text { if }-L \leqslant y<x_{0} \\
\gamma(1-w / k) & \text { if } x_{0}<y \leqslant L\end{cases}\right.
$$

Setting

$$
z:=w X_{y},
$$


we can reformulate problem (3) in terms of $w$ and $z$, considered as functions of $y$ and $t$ :

$$
\begin{cases}w_{t}=\frac{w^{2}}{z}\left(\frac{D(y) w \chi^{\prime}(w) w_{y}}{z}\right)_{y}+w h(y, w) & \text { in }(-L, L) \times \mathbb{R}^{+}, \\ z_{t}=z h(y, w) & \text { in }(-L, L) \times \mathbb{R}^{+}, \\ w_{y}( \pm L, t)=0 & \text { if } t>0, \\ w(y, 0)=z(y, 0)=w_{0}(y) & \text { if }-L<y<L .\end{cases}
$$

Indeed, denoting for the moment the variables $(y, t)$ by $(y, \tau)$, we have

$$
\begin{aligned}
w_{\tau} & =w_{x} X_{\tau}+w_{t}=-D w_{x} \chi^{\prime}(w) w_{x}+\left(D w \chi^{\prime}(w) w_{x}\right)_{x}+w h \\
& =w\left(D \chi^{\prime}(w) w_{x}\right)_{x}+w h \\
& =w\left(\frac{D \chi^{\prime}(w) w_{y}}{X_{y}}\right)_{y} \frac{1}{X_{y}}+w h=\frac{w^{2}}{z}\left(\frac{D w \chi^{\prime}(w) w_{y}}{z}\right)_{y}+w h,
\end{aligned}
$$

and

$$
z_{\tau}=w_{\tau} X_{y}+w X_{\tau y}=w\left(\frac{D w \chi^{\prime}(w) w_{y}}{z}\right)_{y}+z h-w\left(\frac{D w \chi^{\prime}(w) w_{y}}{z}\right)_{y}=z h .
$$

To prove Theorem 1.1 we proceed in two steps. First we solve problem (12) assuming that $D$, $h$ and $w_{0}$ are smooth functions of $y$. Then we return to the original variables and eliminate the smoothness conditions on the data.

STEP 1: Solve problem (12) in case of smooth data. We suppose that $D(y), h(y, w)$ and $w_{0}(y)$ are of class $C^{\infty}$. We shall always assume that $h(y, w) \leqslant 0$ if $w \geqslant \alpha_{0}$ for some $\alpha_{0}>0,0<D_{0} \leqslant$ $D(y) \leqslant D_{1}$ and $0<A_{0} \leqslant w_{0}(y) \leqslant B_{0}$ for some constants $D_{0}, D_{1}, A_{0}$ and $B_{0}$. Then problem (12) has a smooth local (with respect to $t$ ) solution, which can be continued to a global solution (for all $t>0$ ) since the maximum principle implies that for all $T>0$ there exist constants $A(T), B$ (which does not depend on $T), a(T)$ and $b(T)$ such that

$$
0<A(T) \leqslant w \leqslant B \quad \text { and } \quad 0<a(T) \leqslant z \leqslant b(T) \quad \text { in }[-L, L] \times[0, T]
$$

(in Step 1 we shall use the convention that bounds and quantitative estimates do not depend on the smoothness of $D, h$ and $w_{0}$, unless specified differently).

Multiplying the equation for $w$ by $z$ and using the equation for $z$ we obtain

$$
\left.\int_{-L}^{L} w z\right|_{t=0} ^{t=T}=2 \iint_{(-L, L) \times(0, T)}\left(-\frac{D w^{2}}{z} \chi^{\prime}(w) w_{y}^{2}+w z h\right) .
$$

In view of 14, this yields a bound for $w_{y}$ in $L^{2}((-L, L) \times(0, T))$.

Set

$$
p(y, t):=\frac{D(y) w \chi^{\prime}(w) w_{y}}{z}=-X_{t}(y, t) .
$$

The bound on $w_{y}$ and 147 imply a bound on $p$ in $L^{2}((-L, L) \times(0, T))$. Hence for any $t_{0}>0$ there exists $\tau \in\left(0, t_{0}\right)$ such that $p(\cdot, \tau)$ is bounded in $L^{2}(-L, L)$ by a constant depending on $t_{0}$ (but again, not on the smoothness of the data). Multiplying the equation for $p$,

$$
p_{t}=\frac{D}{z}\left(\chi^{\prime}(w)\left(\frac{w^{3}}{z} p_{y}+w^{2} h\right)\right)_{y}-p h
$$


by $\frac{z}{D} p$ and using again the equation for $z$, we obtain

$$
\left.\frac{1}{2} \int_{-L}^{L} \frac{z}{D} p^{2}\right|_{t=\tau} ^{t=T}=-\iint_{(-L, L) \times(\tau, T)}\left(\chi^{\prime}(w)\left(\frac{w^{3}}{z} p_{y}^{2}+w^{2} h p_{y}\right)+\frac{z h}{2 D} p^{2}\right) .
$$

By Hölder's and Young's inequalities,

$$
\left|\iint_{(-L, L) \times(\tau, T)} \chi^{\prime}(w) w^{2} h p_{y}\right| \leqslant \frac{1}{2} \iint_{(-L, L) \times(\tau, T)} \chi^{\prime}(w)\left(\frac{w^{3}}{z} p_{y}^{2}+w z h^{2}\right),
$$

and hence (using (14) and the arbitrariness of $t_{0}>0$ ) we obtain bounds of $w_{y}$ in $L_{\mathrm{loc}}^{\infty}\left((0, T] ; L^{2}(-L, L)\right)$ and of $p=D w \chi^{\prime}(w) w_{y} / z$ in $L_{\mathrm{loc}}^{2}\left((0, T] ; H^{1}(-L, L)\right) \subset$ $L_{\text {loc }}^{2}((0, T] ; C([-L, L]))$. In particular $w_{y}$ is bounded in $L_{\mathrm{loc}}^{\infty}([-L, L] \times(0, T])$.

Differentiating the equation for $p$ with respect to $y$ and multiplying by $p_{y} / z+h / w$, we find (using the equations for $w$ and $z$ and the boundary condition $p( \pm L, t)=p_{t}( \pm L, t)=0$ ) that, for all $0<\tau<T$,

$$
\begin{aligned}
\left.\frac{1}{2} \int_{-L}^{L} \frac{p_{y}^{2}}{z}\right|_{t=\tau} ^{t=T}+\left.\int_{-L}^{L} \frac{h p_{y}}{w}\right|_{t=\tau} ^{t=T}+\iint_{(-L, L) \times(\tau, T)}\left(\frac{h}{2 z} p_{y}^{2}-\frac{\partial}{\partial w}\left(\frac{h}{w}\right)\left(\frac{w^{2}}{z} p_{y}^{2}+w h p_{y}\right)\right) \\
=-\iint_{(-L, L) \times(\tau, T)}\left(\frac{D}{z}\left(\chi^{\prime}(w)\left(\frac{w^{3}}{z} p_{y}+w^{2} h\right)\right)_{y}-p h\right)\left(\frac{p_{y}}{z}+\frac{h}{w}\right)_{y} \\
=-\iint_{(-L, L) \times(\tau, T)}\left(\frac{D \chi^{\prime}(w) w^{3}}{z}\left(\frac{p_{y}}{z}+\frac{h}{w}\right)_{y}^{2}+K(y, t)\left(\frac{p_{y}}{z}+\frac{h}{w}\right)_{y}\right),
\end{aligned}
$$

where

$$
K(y, t)=\frac{D\left(w^{3} \chi^{\prime}(w)\right)^{\prime} w_{y} p_{y}}{z^{2}}+\frac{D\left(w^{3} \chi^{\prime}(w)\right)^{\prime} h w_{y}}{w z}-p h .
$$

In view of the bounds obtained before, $p_{y}$ and $K$ are bounded in $L_{\text {loc }}^{2}\left((0, T] ; L^{2}(-L, L)\right)$. Choosing, for fixed $t_{0}>0, \tau \in\left(\frac{1}{2} t_{0}, t_{0}\right)$ such that $p_{y}(\cdot, \tau)$ is bounded in $L^{2}(-L, L)$ by a constant depending on $t_{0}$, we deduce from Hölder's and Young's inequalities that

$$
\frac{1}{4} \int_{-L}^{L} \frac{p_{y}^{2}(\cdot, T)}{z(\cdot, T)}+\frac{1}{2} \iint_{(-L, L) \times(\tau, T)} \frac{D \chi^{\prime}(w) w^{3}}{z}\left(\frac{p_{y}}{z}+\frac{h}{w}\right)_{y}^{2} \leqslant C\left(t_{0}\right)
$$

for some constant $C\left(t_{0}\right)$. So we have found bounds for $p_{y}$ in $L_{\text {loc }}^{\infty}\left((0, T] ; L^{2}(-L, L)\right)$, $p_{y} / z+h / w$ in $L_{\text {loc }}^{2}\left((0, T] ; H^{1}(-L, L)\right)$, and hence, using the equations for $w$ and $p$, for $w_{t}$ in $L_{\mathrm{loc}}^{\infty}\left((0, T] ; L^{2}(-L, L)\right)$ and $p_{t}$ in $L_{\mathrm{loc}}^{2}([-L, L] \times(0, T])$.

Summarizing, in case of smooth data and with the assumptions listed at the beginning of Step 1, problem (12) has a smooth and globally defined solution which satisfies a priori estimates for $w_{y}$ (in $\left.L_{\mathrm{loc}}^{\infty}([-L, L] \times(0, T])\right), p=D w \chi^{\prime}(w) w_{y} / z$ (in $\left.L_{\mathrm{loc}}^{\infty}\left((0, T] ; H^{1}(-L, L)\right)\right), w_{t}$ (in $\left.L_{\mathrm{loc}}^{\infty}\left((0, T] ; L^{2}(-L, L)\right)\right), p_{t}$ (in $\left.L_{\mathrm{loc}}^{2}([-L, L] \times(0, T])\right)$, and $z_{t}\left(\right.$ in $\left.L^{\infty}((-L, L) \times(0, T))\right)$, which do not depend on the smoothness of the data. The same holds for the estimates in (14).

Finally we observe (although we shall not use it in what follows) that $w$ and $z$ satisfy

$$
\left(\frac{z}{w}\right)_{t}+\left(\frac{D w \chi^{\prime}(w) w_{y}}{z}\right)_{y}=0
$$


Indeed,

$$
\left(\frac{z}{w}\right)_{t}=\frac{z_{t}}{w}-\frac{z}{w^{2}} w_{t}=-\left(\frac{D w \chi^{\prime}(w) w_{y}}{z}\right)_{y}
$$

Alternatively, this equation can be obtained from differentiating the equality $X_{t}+p=0$ with respect to $y$.

STEP 2: Back to the original variables. We approximate $D(y), h(y, w)$ and $w_{0}(y)$ by smooth functions $D_{\varepsilon}, h_{\varepsilon}$ and $w_{0 \varepsilon}$. We shall assume that

$$
\begin{aligned}
D_{\varepsilon}(y) & = \begin{cases}1 & \text { if }-L \leqslant y \leqslant x_{0}-\varepsilon \\
d & \text { if } x_{0}+\varepsilon \leqslant y \leqslant L\end{cases} \\
h_{\varepsilon}(y, w) & = \begin{cases}1-w & \text { if }-L \leqslant y \leqslant x_{0}-\varepsilon \\
\gamma(1-w / k) & \text { if } x_{0}+\varepsilon \leqslant y \leqslant L .\end{cases}
\end{aligned}
$$

We apply the bounds obtained in Step 1 to the solutions $\left(w_{\varepsilon}(y, t), z_{\varepsilon}(y, t)\right)$ of the approximating problems. All bounds are uniform in $\varepsilon$. In particular the uniform bounds on $w_{\varepsilon}$ and $p_{\varepsilon}$ imply that these functions are precompact in $C\left([-L, L] \times\left[t_{0}, T\right]\right)$ for all $0<t_{0}<T$. Hence it follows from a standard diagonal procedure that there exist a sequence $\varepsilon_{n} \rightarrow 0$ as $n \rightarrow \infty$ and functions $w, p \in C([-L, L] \times(0, \infty))$ such that for all $0<t_{0}<T$,

$$
w_{n}:=w_{\varepsilon_{n}} \rightarrow w \quad \text { and } \quad p_{n}:=p_{\varepsilon_{n}} \rightarrow p \quad \text { in } C\left([-L, L] \times\left[t_{0}, T\right]\right) .
$$

Define $X_{n}$ in $[-L, L] \times[0, \infty)$ by $X_{n t}=-p_{n}$ and $X_{n}(y, 0)=y$. Then there exists a function $X \in C^{0,1}([-L, L] \times(0, \infty))$ such that $X_{n} \rightarrow X$ in $C^{0,1}\left([-L, L] \times\left[t_{0}, T\right]\right)$ for all $0<t_{0}<T$. Obviously $X_{t}=-p$ in $[-L, L] \times \mathbb{R}^{+}$. Since $0<\delta_{1} \leqslant X_{n y} \leqslant \delta_{2}$ for some constants $\delta_{1}, \delta_{2}$ which do not depend on $n$, the transformations $x=X_{n}(y, t)$ and $x=X(y, t)$ are invertible for all fixed $t>0$, and the inverse function $X_{n}^{-1}(x, t)$ satisfies $0<\delta_{2}^{-1} \leqslant\left(X_{n}^{-1}\right)_{x}(x, t) \leqslant \delta_{1}^{-1}$ for all $x \in[-L, L]$ and $t>0$.

Setting

$$
\zeta(t)=X\left(x_{0}, t\right) \text { for } t>0,
$$

we define the sets $\mathcal{P}_{u}$ and $\mathcal{P}_{v}$ as in Theorem 1.1 and set

$$
\begin{aligned}
& u_{n}(x, t)=\left\{\begin{array}{ll}
w_{n}\left(X_{n}^{-1}(x, t), t\right) & \text { if }(x, t) \in \mathcal{P}_{u}, \\
0 & \text { if }(x, t) \in \mathcal{P}_{v},
\end{array} \quad v_{n}(x, t)= \begin{cases}0 & \text { if }(x, t) \in \mathcal{P}_{u}, \\
w_{n}\left(X_{n}^{-1}(x, t), t\right) & \text { if }(x, t) \in \mathcal{P}_{v},\end{cases} \right. \\
& u(x, t)=\left\{\begin{array}{ll}
w\left(X^{-1}(x, t), t\right) & \text { if }(x, t) \in \mathcal{P}_{u}, \\
0 & \text { if }(x, t) \in \mathcal{P}_{v},
\end{array} \quad \text { if }(x, t) \in \mathcal{P}_{u},\right.
\end{aligned}
$$

Arguing similarly to (13) and using (15), in any compact subset $K_{u}$ (resp. $K_{v}$ ) of $\mathcal{P}_{u}$ (resp. $\mathcal{P}_{v}$ ) we find that for large enough values of $n, u_{n}$ and $v_{n}$ satisfy the uniformly parabolic equations

$$
u_{n t}=\left(u_{n} \chi^{\prime}\left(u_{n}\right) u_{n x}\right)_{x}+u_{n}\left(1-u_{n}\right) \quad \text { in } K_{u}
$$

and

$$
v_{n t}=d\left(v_{n} \chi^{\prime}\left(v_{n}\right) v_{n x}\right)_{x}+\gamma v_{n}\left(1-\frac{v_{n}}{k}\right) \text { in } K_{v} .
$$


Hence it follows from standard theory that $u \in C^{2,1}\left(\mathcal{P}_{u}\right), v \in C^{2,1}\left(\mathcal{P}_{v}\right)$,

$$
\begin{array}{ll}
u_{t}=\left(u \chi^{\prime}(u) u_{x}\right)_{x}+u(1-u) & \text { in } \mathcal{P}_{u}, \\
v_{t}=d\left(v \chi^{\prime}(v) v_{x}\right)_{x}+\gamma v(1-v / k) & \text { in } \mathcal{P}_{v} .
\end{array}
$$

In addition $u(\cdot, t) \rightarrow w_{0}$ in $L^{1}\left(-L, x_{0}\right)$ and $v(\cdot, t) \rightarrow w_{0}$ in $L^{1}\left(x_{0}, L\right)$ as $t \rightarrow 0^{+}$.

In order to complete the proof of Theorem 1.1, it remains to show that $u, v$ and $\zeta$ have all required properties concerning the interface $x=\zeta(t)$. These properties follow almost at once from the continuity of the functions $w(y, t)$ and $p(y, t)$ across the vertical line $y=x_{0}$. We omit the details.

\section{Equal dispersal velocities}

In this section we prove Theorem 1.2, i.e. the existence of a solution of problem 3 if $d=1$, without assuming initial segregation of $u$ and $v$.

If $d=1$ the equation for $w=u+v$ is particularly simple: adding the equations for $u$ and $v$ and defining

$$
r(x, t)=u(x, t) / w(x, t)
$$

we obtain

$$
w_{t}=\left(w \chi^{\prime}(w) w_{x}\right)_{x}+F(w, r), \quad r_{t}=r_{x} \chi^{\prime}(w) w_{x}+G(w, r),
$$

where we have set

$$
\begin{aligned}
f(u, v):=u(1-u-\alpha v), \quad g(u, v):=\gamma v(1-\beta u-v / k) & \text { for } u, v \geqslant 0, \\
F(w, r):=f(r w,(1-r) w)+g(r w,(1-r) w) & \text { for } w \geqslant 0,0 \leqslant r \leqslant 1 \\
G(w, r):=r(1-r)\left(\frac{f(r w,(1-r) w)}{r w}-\frac{g(r w,(1-r) w)}{(1-r) w}\right) & \text { for } w \geqslant 0,0 \leqslant r \leqslant 1 .
\end{aligned}
$$

The equations for $w$ and $r$ are completed by a boundary condition for $w$ and initial conditions for $w$ and $r$ :

$$
\begin{aligned}
& w_{x}( \pm L, t)=0 \quad \text { for } t>0 \\
& w(x, 0)=w_{0}(x):=u_{0}(x)+v_{0}(x) \quad \text { and } \quad r(x, 0)=r_{0}(x):=\frac{u_{0}(x)}{w_{0}(x)} \quad \text { for }-L<x<L .
\end{aligned}
$$

Let $\varepsilon>0$. We consider the approximating problem

$$
\begin{cases}w_{t}=\left(w \chi^{\prime}(w) w_{x}\right)_{x}+F(w, r) & \text { for }-L<x<L, t>0, \\ r_{t}=r_{x} \chi^{\prime}(w) w_{x}+G(r, w)+\varepsilon r_{x x} & \text { for }-L<x<L, t>0, \\ w_{x}( \pm L, t)=r_{x}( \pm L, t)=0 & \text { for } t>0, \\ w(x, 0)=w_{0 \varepsilon}(x), \quad r(x, 0)=r_{0 \varepsilon}(x) & \text { for }-L<x<L .\end{cases}
$$

Here

$w_{0 \varepsilon}, r_{0 \varepsilon} \in C^{\infty}([-L, L]), \quad w_{0 \varepsilon} \rightarrow w_{0}$ in $C^{1}([-L, L])$ as $\varepsilon \rightarrow 0$,

$w_{0 \varepsilon}^{\prime}$ is uniformly Hölder continuous in $[-L, L]$, 


$$
\begin{aligned}
& w_{0 \varepsilon} \geqslant \delta_{0}:=\min _{[-L, L]} w_{0}>0, \quad w_{0 \varepsilon} \leqslant \delta_{1}:=\max _{[-L, L]} w_{0}>0, \quad \int_{-L}^{L} w_{0 \varepsilon}=\int_{-L}^{L} w_{0}, \\
& r_{0 \varepsilon} \rightarrow r_{0} \quad \text { in } L^{2}(-L, L) \text { and a.e. in }(-L, L) \text { as } \varepsilon \rightarrow 0, \\
& 0 \leqslant r_{0 \varepsilon} \leqslant 1 \text { in }[-L, L], \quad \int_{-L}^{L}\left|r_{0 \varepsilon}^{\prime}\right| \rightarrow \operatorname{TV}\left(r_{0}\right) \text { as } \varepsilon \rightarrow 0, \quad \varepsilon \int_{-L}^{L}\left(r_{0 \varepsilon}^{\prime}\right)^{2} \leqslant C,
\end{aligned}
$$

where TV indicates total variation.

LEMMA 3.1 Let $w_{0 \varepsilon}$ and $r_{0 \varepsilon}$ satisfy the hypotheses listed above and let, for all $0<\varepsilon \leqslant 1,\left(w_{\varepsilon}, r_{\varepsilon}\right)$ be a classical solution of problem (16) for $0 \leqslant t \leqslant T$. Then

(i) $0 \leqslant r_{\varepsilon} \leqslant 1$ in $[-L, L] \times[0, T]$ and there exist constants $0<C_{1} \leqslant C_{2}$ which do not depend on $\varepsilon$ and $T$ such that $C_{1} \leqslant w_{\varepsilon} \leqslant C_{2}$ in $[-L, L] \times[0, T]$;

(ii) $\chi\left(w_{\varepsilon}\right)_{x x}$ and $w_{\varepsilon t}$ are equibounded in $L^{2}((-L, L) \times(0, T))$;

(iii) $w_{\varepsilon}$ and $w_{\varepsilon x}$ are uniformly Hölder continuous in $[-L, L] \times[0, T]$;

(iv) $r_{\varepsilon}$ is equibounded in $L^{\infty}(0, T ; B V(-L, L))$;

(v) $r_{\varepsilon}$ is equibounded in $B V((-L, L) \times(0, T))$.

Proof. (i) Applying the comparison principle to the equation for $r_{\varepsilon}$, it follows at once that $0 \leqslant$ $r_{\varepsilon} \leqslant 1$ in $[-L, L] \times[0, \infty)$. Since $f(r w,(1-r) w)<0(>0)$ for all $0 \leqslant r \leqslant 1$ if $w>$ $1 / \min \{1, \alpha\}$ (respectively $w<1 / \max \{1, \alpha\}$ ), and $g(r w,(1-r) w)<0(>0)$ for all $0 \leqslant r \leqslant 1$ if $w>1 / \min \{1 / k, \beta\}$ (respectively $w<1 / \max \{1 / k, \beta\}$ ), it follows from the comparison principle applied to the equation for $w_{\varepsilon}$ that $C_{1} \leqslant w_{\varepsilon} \leqslant C_{2}$ in $[-L, L] \times[0, \infty)$, where

$$
\begin{aligned}
& C_{1}=\min \left\{\delta_{0}, \frac{1}{\max \{1, \alpha\}}, \frac{1}{\max \{1 / k, \beta\}}\right\}, \\
& C_{2}=\max \left\{\delta_{1}, \frac{1}{\min \{1, \alpha\}}, \frac{1}{\min \{1 / k, \beta\}}\right\} .
\end{aligned}
$$

(ii)-(iii) Multiplying the equation for $w_{\varepsilon}$ by $\chi^{\prime}\left(w_{\varepsilon}\right) \chi\left(w_{\varepsilon}\right)_{x x}$, integrating by parts, using Hölder's and Young's inequalities and omitting the subscripts $\varepsilon$, we obtain

$$
\begin{aligned}
\left.\frac{1}{2} \int_{-L}^{L} \chi(w)_{x}^{2}\right|_{0} ^{T} & =-\int_{0}^{T} \int_{-L}^{L}\left(w \chi^{\prime}(w) \chi(w)_{x x}^{2}+\chi(w)_{x}^{2} \chi(w)_{x x}+\chi^{\prime}(w) F(w, r) \chi(w)_{x x}\right) \\
& \leqslant C_{3} \int_{0}^{T} \int_{-L}^{L}\left|\chi(w)_{x x}\right|-C_{1} \int_{0}^{T} \int_{-L}^{L} \chi(w)_{x x}^{2} \\
& \leqslant-\frac{1}{2} C_{1} \int_{0}^{T} \int_{-L}^{L} \chi(w)_{x x}^{2}+C_{4} T
\end{aligned}
$$

where $C_{3}$ and $C_{4}$ are positive constants which do not depend on $\varepsilon$ and $T$. Hence $\chi\left(w_{\varepsilon}\right)_{x x}$ is equibounded in $L^{2}((-L, L) \times(0, T))$ and $w_{\varepsilon}$ is equibounded in $L^{\infty}\left([0, T] ; H^{1}(-L, L)\right)$. In particular $w_{\varepsilon}$ is uniformly Hölder continuous with respect to $x$, and hence, by standard results ([6], [7]), also with respect to $t$.

Set

$$
s_{\varepsilon}(x, t):=\int_{1}^{w_{\varepsilon}(x, t)} w \chi^{\prime}(w) \mathrm{d} w .
$$


Then $s_{\varepsilon}$ satisfies the equation $s_{t}=w_{\varepsilon} \chi^{\prime}\left(w_{\varepsilon}\right) s_{x x}+w_{\varepsilon} \chi^{\prime}\left(w_{\varepsilon}\right) F\left(w_{\varepsilon}, r_{\varepsilon}\right)$. It follows from the techniques used in [8, Chapter IV, Corollary of Theorem 9.1] that $s_{\varepsilon x}$, and thus also $w_{\varepsilon x}$, is uniformly Hölder continuous in $[-L, L] \times[0, T]$. Finally, the equiboundedness of $w_{\varepsilon t}$ in $L^{2}((-L, L) \times(0, T))$ follows from the equation for $w_{\varepsilon}$ and the estimates for $\chi\left(w_{\varepsilon}\right)_{x x}$ and $w_{\varepsilon x}$.

(iv) Omitting the subscripts $\varepsilon$, we observe that $r_{x}$ satisfies the equation

$$
\left(r_{x}\right)_{t}=\left(r_{x} \chi(w)_{x}\right)_{x}+G_{w} w_{x}+G_{r} r_{x}+\varepsilon\left(r_{x}\right)_{x x}
$$

where $G_{w}$ and $G_{r}$ are bounded in bounded subsets of $[0, \infty) \times[0,1]$. Multiplying the equation, for fixed $\delta>0$, by $r_{x} / \sqrt{\delta+r_{x}^{2}}$ and integrating by parts we obtain

$$
\begin{aligned}
\left.\int_{-L}^{L} \sqrt{\delta+r_{x}^{2}}\right|_{0} ^{T} \leqslant & -\int_{0}^{T} \int_{-L}^{L} \delta \frac{r_{x x}}{\left(\delta+r_{x}^{2}\right)^{3 / 2}} r_{x} \chi(w)_{x} \\
& +\int_{0}^{T} \int_{-L}^{L}\left(\left|G_{w} w_{x}\right|+\left|G_{r} r_{x}\right|-\varepsilon \delta \frac{r_{x x}^{2}}{\left(\delta+r_{x}^{2}\right)^{3 / 2}}\right) \\
\leqslant & C \int_{0}^{T} \int_{-L}^{L}\left(\left|w_{x}\right|+\left|r_{x}\right|\right)+\int_{0}^{T} \int_{-L}^{L} \delta \chi(w)_{x}\left(\frac{1}{\sqrt{\delta+r_{x}^{2}}}\right)_{x},
\end{aligned}
$$

where the constant $C$ does not depend on $\varepsilon$ and $\delta$. Integration by parts implies that the latter term can be estimated by

$$
\int_{0}^{T} \int_{-L}^{L} \frac{\delta\left|\chi(w)_{x x}\right|}{\sqrt{\delta+r_{x}^{2}}} \leqslant \sqrt{\delta} \int_{0}^{T} \int_{-L}^{L}\left|\chi(w)_{x x}\right|
$$

which vanishes as $\delta \rightarrow 0$. It follows from Gronwall's lemma that, in the limit for vanishing $\delta$, $\sup _{[0, T]} \int_{-L}^{L}\left|r_{\varepsilon x}(x, t)\right| \mathrm{d} x$ is uniformly bounded.

(v) In view of (iv) we must prove that $r_{\varepsilon t}$ is equibounded in $L_{1}((-L, L) \times(0, T))$. Omitting the subscripts $\varepsilon$, it follows from the equation for $r$ that there exists a constant $C$ which does not depend on $\varepsilon$ and $T$ such that

$$
\int_{0}^{T} \int_{-L}^{L}\left|r_{t}\right| \leqslant I_{1}+I_{2}+C:=\int_{0}^{T} \int_{-L}^{L}\left|\chi(w)_{x}\right| \cdot\left|r_{x}\right|+\varepsilon \int_{0}^{T} \int_{-L}^{L}\left|r_{x x}\right|+C .
$$

Since $w_{x}$ is equibounded in $[-L, L] \times[0, T]$, (iv) implies that $I_{1}$ is equibounded. It remains to prove that also $I_{2}$ is equibounded.

We multiply the equation for $r$ by $\varepsilon r_{x x}$ and integrate by parts:

$$
\begin{aligned}
-\left.\frac{1}{2} \int_{-L}^{L} \varepsilon r_{x}^{2}\right|_{0} ^{T} & =J_{1}+J_{2}+J_{3} \\
& :=-\frac{1}{2} \int_{0}^{T} \int_{-L}^{L} \varepsilon r_{x}^{2} \chi(w)_{x x}+\int_{0}^{T} \int_{-L}^{L} \varepsilon r_{x x} G+\varepsilon^{2} \int_{0}^{T} \int_{-L}^{L} r_{x x}^{2} .
\end{aligned}
$$

We observe that $\varepsilon \int_{-L}^{L}\left(r_{\varepsilon 0}^{\prime}\right)^{2}$ is equibounded and that, by Hölder's and Young's inequalities, $\left|J_{2}\right| \leqslant$ $\frac{1}{2} J_{3}+C$ for some constant $C$ which does not depend on $\varepsilon$. It remains to prove that

$$
\left|J_{1}\right| \leqslant \frac{1}{4} J_{3}+C
$$


for some constant $C$ which does not depend on $\varepsilon$. Indeed, (17) implies the equiboundedness of $J_{3}$, i.e. the equiboundedness of $\varepsilon r_{\varepsilon x x}$ in $L^{2}((-L, L) \times(0, T))$ and hence in $L^{1}((-L, L) \times(0, T))$.

Let $\mu>0$ to be determined below. Then

$$
\left|J_{1}\right| \leqslant \mu \int_{0}^{T} \int_{-L}^{L} \varepsilon^{2} r_{x}^{4}+\frac{1}{16 \mu} \int_{0}^{T} \int_{-L}^{L} \chi(w)_{x x}^{2} \leqslant \mu \int_{0}^{T} \int_{-L}^{L} \varepsilon^{2} r_{x}^{4}+\frac{C}{\mu}
$$

for some constant $C$ which does not depend on $\varepsilon$. Integration by parts yields

$$
\begin{aligned}
\mu \int_{0}^{T} \int_{-L}^{L} \varepsilon^{2} r_{x}^{4} & =\mu \int_{0}^{T} \int_{-L}^{L} \varepsilon^{2} r_{x} r_{x}^{3}=-3 \mu \int_{0}^{T} \int_{-L}^{L} \varepsilon^{2} r_{x x} r_{x}^{2} r \\
& \leqslant \frac{3 \mu}{4} \int_{0}^{T} \int_{-L}^{L} \varepsilon^{2} r_{x}^{4}+3 \mu \int_{0}^{T} \int_{-L}^{L} \varepsilon^{2} r_{x x}^{2},
\end{aligned}
$$

whence

$$
\mu \int_{0}^{T} \int_{-L}^{L} \varepsilon^{2} r_{x}^{4} \leqslant 12 \mu \int_{0}^{T} \int_{-L}^{L} \varepsilon^{2} r_{x x}^{2}=12 \mu J_{3} \leqslant \frac{1}{4} J_{3}
$$

if we choose $48 \mu \leqslant 1$. Hence we have obtained (17) and the proof of Lemma 3.1 is complete.

The following result follows from standard PDE theory:

LEMMA 3.2 Let $w_{0 \varepsilon}$ and $r_{0 \varepsilon}$ satisfy the conditions of Lemma 3.1. Then for all $T>0$ problem (16) has a unique classical solution, $\left(w_{\varepsilon}, r_{\varepsilon}\right)$, defined for all $t \geqslant 0$.

Before passing to the limit $\varepsilon \rightarrow 0$ we study the "characteristics" of the approximating problems: let $X_{\varepsilon}(y, t)$ be defined by

$$
\begin{cases}X_{\varepsilon t}(y, t)=-\chi^{\prime}\left(w_{\varepsilon}\right) w_{\varepsilon x} & \text { if }-L \leqslant y \leqslant L, t>0 \\ X_{\varepsilon}(y, 0)=y & \text { if }-L \leqslant y \leqslant L\end{cases}
$$

Observe that for all $t>0$,

$$
-L \leqslant X_{\varepsilon}\left(y_{1}, t\right)<X_{\varepsilon}\left(y_{2}, t\right) \leqslant L \quad \text { if }-L \leqslant y_{1}<y_{2} \leqslant L
$$

and

$$
\frac{\mathrm{d}}{\mathrm{d} t} \int_{X_{\varepsilon}\left(y_{1}, t\right)}^{X_{\varepsilon}\left(y_{2}, t\right)} w_{\varepsilon}(x, t) \mathrm{d} x=\int_{X_{\varepsilon}\left(y_{1}, t\right)}^{X_{\varepsilon}\left(y_{2}, t\right)} F\left(w_{\varepsilon}(x, t), r_{\varepsilon}(x, t)\right) \mathrm{d} x \quad \text { if }-L \leqslant y_{1}<y_{2} \leqslant L .
$$

Hence there exists a constant $C>0$ which does not depend on $\varepsilon, y_{1}$ and $y_{2}\left(-L \leqslant y_{1}<y_{2} \leqslant L\right)$ such that for all $t>0$,

$$
\left|\frac{\mathrm{d}}{\mathrm{d} t} \int_{X_{\varepsilon}\left(y_{1}, t\right)}^{X_{\varepsilon}\left(y_{2}, t\right)} w_{\varepsilon}(x, t) \mathrm{d} x\right| \leqslant C \int_{X_{\varepsilon}\left(y_{1}, t\right)}^{X_{\varepsilon}\left(y_{2}, t\right)} w_{\varepsilon}(x, t) \mathrm{d} x .
$$

Since $X_{\varepsilon}\left(y_{2}, 0\right)-X_{\varepsilon}\left(y_{1}, 0\right)=y_{2}-y_{1}$, it follows from Lemma 3.1 i) and 21) that for all $T>0$ there exist constants $0<k_{T} \leqslant K_{T}$ such that

$$
k_{T}\left(y_{2}-y_{1}\right) \leqslant X_{\varepsilon}\left(y_{2}, t\right)-X_{\varepsilon}\left(y_{1}, t\right) \leqslant K_{T}\left(y_{2}-y_{1}\right) \quad \text { if }-L \leqslant y_{1}<y_{2} \leqslant L, 0 \leqslant t \leqslant T .
$$

So we have proved the following result: 
LEMmA 3.3 Let $T>0$ and let $X_{\varepsilon}$ be the solution of 18 . Then there exist constants $0<k_{T} \leqslant K_{T}$ such that

$$
0<k_{T} \leqslant X_{\varepsilon y} \leqslant K_{T} \text { in }[-L, L] \times[0, T]
$$

and

$$
X_{\varepsilon} \text { is uniformly bounded in } C^{1}([-L, L] \times[0, T]) .
$$

In addition $X_{\varepsilon t}$ is uniformly Hölder continuous in $[-L, L] \times[0, T]$. (22).

The latter estimate follows from the equation for $X_{\varepsilon}$, the Hölder continuity of $w_{\varepsilon x}(x, t)$ and

Now we are ready to pass to the limit for vanishing $\varepsilon$ : by Lemmas $3.1+3.3$, there exist a sequence $\varepsilon_{n} \rightarrow 0$ and functions $w(x, t), r(x, t)$ and $X(y, t)$ such that $w_{\varepsilon_{n}}, r_{\varepsilon_{n}}$ and $X_{\varepsilon_{n}}$, which we shall denote by $w_{n}, r_{n}$ and $X_{n}$, satisfy, for all $T>0$,

$$
\begin{aligned}
& X \text { is defined and Lipschitz continuous in }[-L, L] \times[0, T], \\
& X_{t} \text { is Hölder continuous in }[-L, L] \times[0, T], \\
& X_{n} \rightarrow X \text { uniformly in }[-L, L] \times[0, T] \text { as } n \rightarrow \infty, \\
& w \text { and } w_{x} \text { are defined and Hölder continuous in }[-L, L] \times[0, T], \\
& w_{t}, \chi(w)_{x x} \in L^{2}((-L, L) \times(0, T)), \\
& w_{n} \rightarrow w \text { and } w_{n x} \rightarrow w_{x} \text { uniformly in }[-L, L] \times[0, T] \text { as } n \rightarrow \infty, \\
& X_{t}=-\chi(w)_{x}(X, t) \text { in }[-L, L] \times[0, T], \\
& r \in B V((-L, L) \times(0, T)) \cap L^{\infty}(0, T ; B V(-L, L)), \\
& r_{n} \rightarrow r \text { a.e. in }(-L, L) \times(0, T) \text { as } n \rightarrow \infty, \\
& r_{n} \rightarrow r \text { in } L^{q}((-L, L) \times(0, T))(1 \leqslant q<\infty) \text { as } n \rightarrow \infty, \\
& w_{t}=\left(w \chi^{\prime}(w) w_{x}\right)_{x}+F(w, r) \text { in } L^{2}((-L, L) \times(0, T)) \\
& r_{t}=\left(r \chi^{\prime}(w) w_{x}\right)_{x}-r \chi(w)_{x x}+G(w, r) \text { weakly, }
\end{aligned}
$$

i.e., for any smooth function $\psi(x, t)$,

$$
\begin{aligned}
\int_{-L}^{L} r_{0}(x) \psi(x, 0)-\int_{-L}^{L} r(x, T) \psi & (x, T)+\int_{0}^{T} \int_{-L}^{L} r \psi_{t} \\
& =\int_{0}^{T} \int_{-L}^{L}\left(r \chi(w)_{x} \psi_{x}+r \chi(w)_{x x} \psi-G(w, r) \psi\right) .
\end{aligned}
$$

To obtain the latter formula we have used that

$$
r_{n} \rightarrow r \in C\left([0, T] ; L^{1}(L, L)\right) \quad \text { as } n \rightarrow \infty,
$$

which follows easily from the equiboundedness of $r_{n x}$ in $L^{\infty}\left([0, T] ; L^{1}(-L, L)\right)$ and the equiHölder continuity of $w_{n x}$ : for $0<h<1$,

$$
\int_{-L}^{L}\left|r_{n}(x, t+h)-r_{n}(x, t)\right| \leqslant \int_{t}^{t+h} \int_{-L}^{L}\left|r_{n x} \chi^{\prime}\left(w_{n}\right) w_{n x}+F\left(w_{n}, r_{n}\right)\right| \leqslant K h,
$$

where $K$ is a constant which does not depend on $n$ and $h$. 
Setting

$$
u:=r w \quad \text { and } \quad v:=w-u,
$$

we claim that $u$ and $v$ have all properties listed in Theorem 1.2 Indeed, having in mind the properties (23)-(35) of $w$ and $r$, it remains to prove (10) and (11). Here we shall only prove (10).

Let $\varphi \in C^{\infty}([-L, L] \times[0, T])$. Clearly $[35)$ holds also for $\psi:=\varphi w$ and we obtain

$$
\begin{aligned}
\int_{-L}^{L} u_{0}(x) \varphi(x, 0) & -\int_{-L}^{L} u(x, T) \varphi(x, T)+\int_{0}^{T} \int_{-L}^{L} u \varphi_{t} \\
+ & \int_{0}^{T} \int_{-L}^{L} \frac{u \varphi}{w}\left(w \chi(w)_{x x}+\chi^{\prime}(w) w_{x}^{2}+F\left(\frac{u}{w}, w\right)\right) \\
& =\int_{0}^{T} \int_{-L}^{L}\left(\frac{u \chi(w)_{x}}{w}\left(\varphi_{x} w+\varphi w_{x}\right)+u \chi(w)_{x x} \varphi-w G\left(\frac{u}{w}, w\right) \varphi\right) .
\end{aligned}
$$

Hence

$$
\begin{aligned}
\int_{-L}^{L} u_{0}(x) \varphi(x, 0)- & \int_{-L}^{L} u(x, T) \varphi(x, T)+\int_{0}^{T} \int_{-L}^{L} u \varphi_{t} \\
& =\int_{0}^{T} \int_{-L}^{L} u \chi(w)_{x} \varphi_{x}-\int_{0}^{T} \int_{-L}^{L}\left(\frac{u}{w} F\left(\frac{u}{w}, w\right)+w G\left(\frac{u}{w}, w\right)\right) \varphi \\
& =\int_{0}^{T} \int_{-L}^{L}\left(u \chi(w)_{x} \varphi_{x}-f(u, v) \varphi\right),
\end{aligned}
$$

and we have found $[10]$.

\section{Acknowledgements}

We would like to thank Luigi Preziosi for a fruitful discussion about contact inhibition problems from a biological point of view. This study was supported by KAKENHI S (No.18104002) and the Global COE program (G14) "Formation and Development of Mathematical Sciences based on Modeling and Analysis".

\section{REFERENCES}

1. Abercrombie, M. Contact inhibition in tissue culture. In Vitro 6 (1970), 128-142.

2. Bertsch, M., Gurtin, M. E., \& Hilhorst, D. On a degenerate diffusion equation of the form $c(z)_{t}=\varphi\left(z_{x}\right)_{x}$ with applicaqtion to population dynamics. J. Differential Equations 30 (1987), 56-89. Zbl 0624.35049 MR 0878252

3. Bertsch, M., Gurtin, M. E., \& Hilhorst, D. On interacting populations that disperse to avoid crowding: the case of equal dispersal velocities. Nonlinear Anal. 11 (1987), 493-499. Zbl 0646.92018 MR 0887658

4. Bertsch, M., Gurtin, M. E., Hilhorst, D., \& Peletier, L. A. On interacting populations that disperse to avoid crowding: preservation of segregation. J. Math. Biology 23 (1985), 1-13. Zbl 0596.35074 MR 0821681

5. Chaplain, M., Graziano, L., \& Preziosi, L. Mathematical modelling of the loss of tissue compression responsiveness and its role in solid tumour development. Math. Med. Biol. 23 (2006), 197229. Zbl 1098.92037 
6. Gilding, B. H. Hölder continuity of solutions of parabolic equations. J. London Math. Soc. 13 (1976), 103-106. Zbl 0319.35045 MR 0399658

7. KRUZhKov, S. N. Results on the character of the regularity of solutions of parabolic equations and some of their applications. Math. Notes 6 (1969), 517-523.

8. Ladyzhenskaya, O. A., Solonnikov, V. A., \& Ural'Ceva, N. N. Linear and Quasilinear Equations of Parabolic Type. Transl. Math. Monogr. 23, Amer. Math. Soc., Providence, RI (1968). MR 0241822

9. SherRATT, J. A. Wavefront propagation in a competition equation with a new motility term modeling contact inhibition between cell populations. Proc. Roy. Soc. London Ser. A 456 (2000), 2365-2386. Zbl 1005.76098 MR 1796488 